\title{
Hardening features for high manganese steel cores of crosspieces along the way
}

\author{
Irina Shishkina ${ }^{1, *}$ \\ ${ }^{1}$ Russian University of Transport (MIIT), Chasovaya str. 22/2, Moscow, 125190, Russia
}

\begin{abstract}
Herein are established main regularities of steel hardening and defect formation for the working conditions of cross-pieces along the way in order to be able to further reduce the number of samples without losing informativity about the metal functioning of cross-piece. The change in the hardness of the metal along the depth of the cross section at the stage of steady wear occurs with the wear rate of the cross-piece. From the surface of the cross into the depths of the cross section, the hardness of the metal decreases. The rule of change can be considered linear. The generalized results of studies of susceptibility of cross-pieces metal for defects showed that the processes of contact defect occurrence and their expansion on the cross-pieces are fatigue-like.
\end{abstract}

\section{Introduction}

The service life of the crossings of turnouts is determined by the probability of formation of contact-fatigue defects, the intensity of wear, crushing, which depend both on the operating conditions of the products and on the metal properties. The intensity of development of the above defects is associated with the nature of property change of the material on the cores rolling surface during plastic deformation $[1,2]$. Studying the process of hardening and the metal structure on one core during its operation is impossible, since for this it is necessary to cut out samples from it.

Studies of cores hardening are possible on different crosspieces, slightly differing in initial physical and mechanical properties, chemical composition and structure (the presence of residual carbides, size and number of non-metallic inclusions, grain size), as well as operating conditions [3,4]. Reliable quantitative estimates of product properties can only be obtained by statistical analysis of a fairly representative sample. Therefore, the basic patterns of hardening and defect formation of 110G13L steel are established below for the working conditions of the crosspieces along a way in order to be able to further reduce the number of samples without losing information about the work of the crosspiece metal.

\section{Research technique and results}

The study of hardening was performed on ten crosspieces that had wear from 3 to $12 \mathrm{~mm}$.

${ }^{*}$ Corresponding author: shishkinaira@inbox.ru 
After cutting the crosses into samples, a metallographic analysis of the core cross sections of 30-40 mm was performed and graphs of changes in the hardness HRC along the depth of the tip core of crosses with different wear were obtained (Fig. 1). Comparing the graphs of changing the hardness in depth shows that for crosses having different wear, the type of dependencies is almost similar. For most crosses, the scatter of HRC values is in the range of 5 to 10 units.

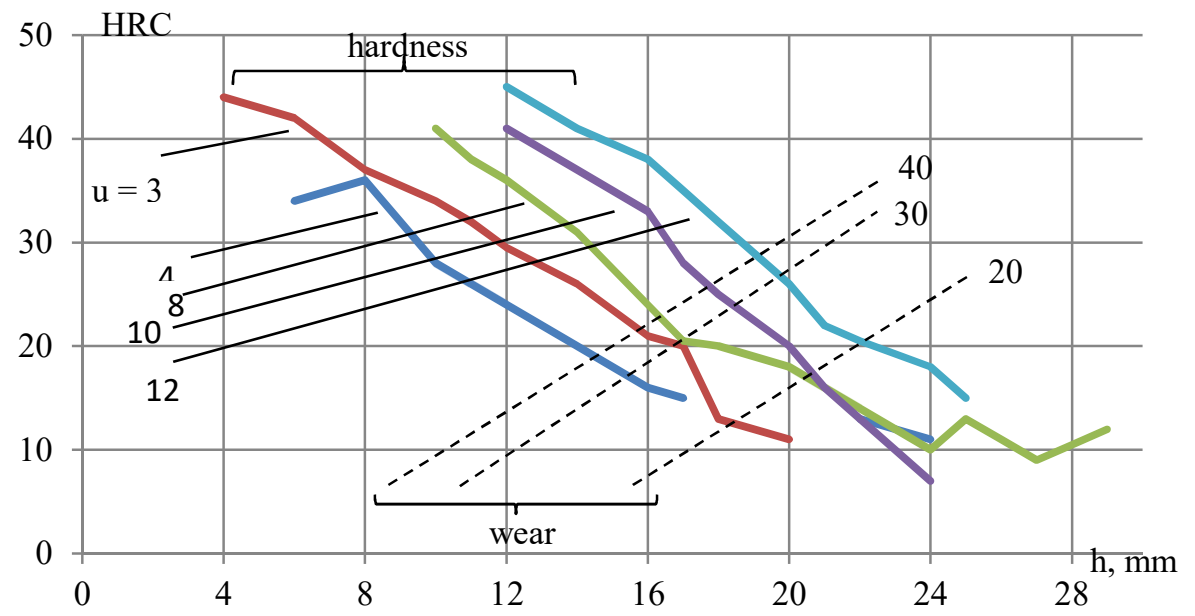

Fig. 1. Change in core metal hardness depending on depth $h$ for different wear of crosspieces (solid lines) and distribution of metal layers with similar hardness (40 HRC, $30 \mathrm{HRC}, 20 \mathrm{HRC}$ ) for crosspieces with different wear (dashed lines)

The coefficients of variation of hardness calculated from the graphs at a depth of 2, 4, 6, 8,10 , and $12 \mathrm{~mm}$ from the surface are respectively $0.09 ; 0.11 ; 0.15 ; 0.15 ; 0.21$ and 0.22 HRC. The average value of the coefficient of variation is $0.15 \mathrm{HRC}$, which is close to similar values for indicators characterizing the mechanical properties, for example, for $\sigma_{\mathrm{B}}$ the coefficient of variation ranges from 0.12 to 0.16 .

Given this fact and the fact that the form of the dependences of hardness on depth for different crosspieces is almost the same, we can conclude that the process of hardening crosspieces in cross sections is ergodic. The ergodicity of hardening makes it possible to replace the sweep of the hardening process of the crosspiece by running time by the cross section scan, that is, in order to find out what hardness will be in any layer of the core of the cross after skipping the amount of load $\Delta \mathrm{T}$, a picture of the hardness distribution is sufficient cross-section at the initial moment, move down by the amount of wear and corresponding to the specified $\Delta \mathrm{T}$, and take the value of hardness at the depth at which this layer is located. All of the above applies to the period after the completion of crushing. Thus, by examining a sample from a crosspiece, it is possible to restore the process of hardening in each of its layers, as well as extrapolating it to the values of wear not yet reached by the cross. To perform such an analysis, it is advisable to use a fixed coordinate system, measuring all the time the position of points from the initial (constant) surfaces of the cross, which is easy to do if its wear is known.

The depth dependences of the hardness depending on the above for the cross section of $30 \mathrm{~mm}$ cores of the crosspieces having different wear, and the isolines of similar hardness constructed with their help in the process of wear, which were constructed using them, are shown in Fig. 1 by dashed lines. The dependence of the depth of the layers with a certain amount of hardness on the wear of the cross is linear, which is another indirect proving of the ergodicity of the process of hardening crosses in the cross section. 
The dependence of depth of the layers with the hardness HRC on wear is:

$$
h_{H R C}=k_{H R C}+u
$$

$k_{H R C}-$ cross hardening depth to a given hardness value; $u$ - cross wear.

In the formula for $h_{H R C}$, the coefficient $u$ before wear of the crosspiece is equal to one. This indicates that the depth of the layer with a certain amount of hardness HRC is linearly related to the wear of the cross.

The coefficient $k_{H R C}$ is a characteristic of the hardening ability of the crosspiece in this section. Knowing its value, one can fully characterize the initial and subsequent positions of metal layers having a given HRC value.

The middle part of dependence of the coefficient $k_{H R C}$ on hardness (Fig. 2) is a straight line. Its points obtained from the graphs in Fig. 1, lie on the line with virtually no scatter. Of particular interest are the intersection points of this straight line with the coordinate axes. In physical sense, $k_{H R C}=0$ on the surface of the cross. Extrapolation of the given dependence gives a value of $47 \mathrm{HRC}$. In fact, for the crosses from which the dependence for $k_{H R C}$ was obtained, the hardness on the surface ranged between 32-47 HRC. This indicates that the $k_{H R C}$ dependence is not linear in the surface layers of the metal. The deviation of the hardening of the linear dependence is observed to a depth of $3 \mathrm{~mm}$. This deviation, as can be seen from the graph, is small and is probably associated with the presence of a large number of microcracks. With an initial hardness of $10 \mathrm{HRC}$, the depth of the hardened layer according to the graph is $16 \mathrm{~mm}$. The actual value of hardening depth for crosses $(17-20 \mathrm{~mm})$ differs little from that obtained from the graph.

Using the graph $k_{H R C}(\mathrm{HRC})$, the surface hardness of the crosspiece can determine the depth of the hardened layer, as well as the position of the layers having the hardness value of interest to us. Moreover, in comparative calculations, you can use the linear dependence over the entire depth of the hardened layer. For more accurate estimates, it is necessary to introduce the corresponding linearity corrections in the extreme layers. You can use another trick: replace the actual dependence with the dependence shown in Fig. 2 dashed lines. In this case, the total depth of the hardened layer will be determined more precisely, and the position of the layers having specific HRC values with an error of up to $1.6 \mathrm{~mm}$.

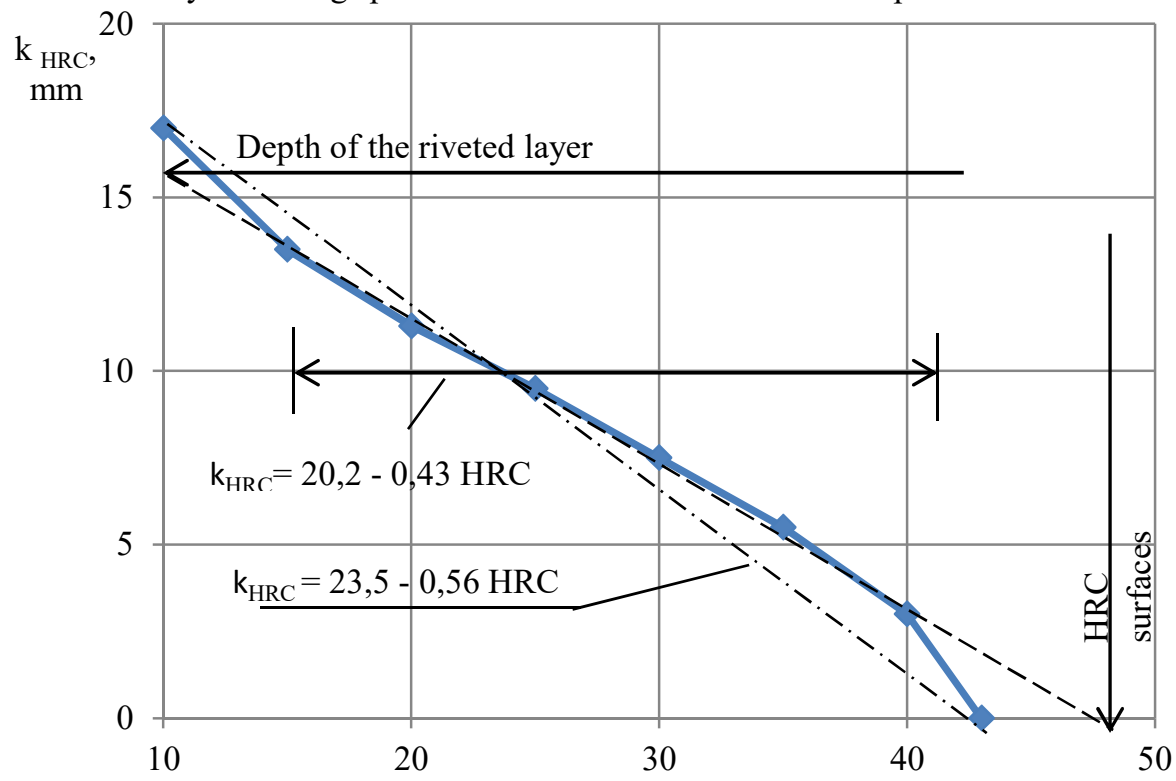

Fig. 2. Dependence of $\mathrm{k}_{\mathrm{HRC}}$ coefficient on hardness HRC 
In the earlier work on the analysis of metal damageability of the crosspieces at various depths from the surface, the number of cracks and their total length were used as the main indicators. As a result of our research, no clear quantitative or qualitative dependencies of these indicators on the wear of the crosspiece or the cargo passed through it were revealed. As in the case of the study of hardening, it is not possible to conduct a study of the dependence of damage indicators on the time or wear on one crosspiece, so studies were conducted on several crossings. The absence of dependences of damageability indicators on running hours and wear was explained by the features of operation of specific crosspieces along the way, the spread of their mechanical characteristics, chemical composition, heat treatment, etc. $[5,6]$.

If we compare the crosspieces with other elements of the track operating under similar conditions, then for them there should be a dependence of the damageability indicators on the operating time $[7,8,9]$.

In contrast to the previous work, an analysis of the indicators of susceptibility of metal to cracks in a fixed coordinate system (from the initial surface) is given below $[10,11,12]$. The advantage of this approach, as already mentioned above, is that it makes it possible to analyze the operation of the same points and layers of metal in the process of wear of the crosspiece, i.e., a change in the indicators of susceptibility to defects in the same metal layers - accumulation in a specific cross-sectional point of the metal indicators of damageability during wear.

Figure 3 shows graphs of the dependence of the number $n$ and the total length $l_{\text {cp }}$ of cracks on the depth of their location for three crosspieces that passed a different amount of cargo and had different wear. When constructing the graphs were shifted so that the depth of the points of all the crosspieces from the initial surface was similar. The graphs show that with increasing wear from 4 to $9 \mathrm{~mm}$ in the same metal layers, the number of cracks increases, then with wear of $12 \mathrm{~mm}$ the number of cracks decreases slightly. This is explained by the fact that during the growth of cracks, part of them can merge, forming cracks of greater length, while the number of cracks will decrease, and their total length will increase $[13,14]$. Therefore, it is more correct to analyze not the number of cracks, but their total length. From the graphs shown in Fig. 3, it can be seen that with an increase in wear from 4 to $12 \mathrm{~mm}$, a monotonic increase in the total length of cracks in the cross section is observed.

According to the graph in Fig. 3, it is possible to obtain indicators of the susceptibility of metal to defects depending on the wear of the crosspieces. One of such indicators is the average crack length at the $i$-th depth, which is determined by the formula

$$
l_{\mathrm{av} i}=\sum l / n,
$$

wherein $\sum l$ and $n$ - total length and number of cracks at $i$-th depth, respectively.

The average length of cracks over the entire cross section can be obtained as the average of the average lengths of cracks in depth, i.e. $\bar{l}_{\mathrm{a} v}=\sum l_{a v i} / j$, wherein $\mathrm{j}$ - number of layers.

The calculation results of the average crack lengths are summarized in table. 1 . The data table. 1 confirm that, with wear in each specific layer at a certain depth, a monotonic increase in the average lengths of cracks is observed [15, 16]. As the crosspieces (passed cargo) wear out, the size of the average crack length along the entire cross section also grows. This indicates that the development of cracks is a consequence of the repeated loads applied, that is, it has a fatigue-like character. 


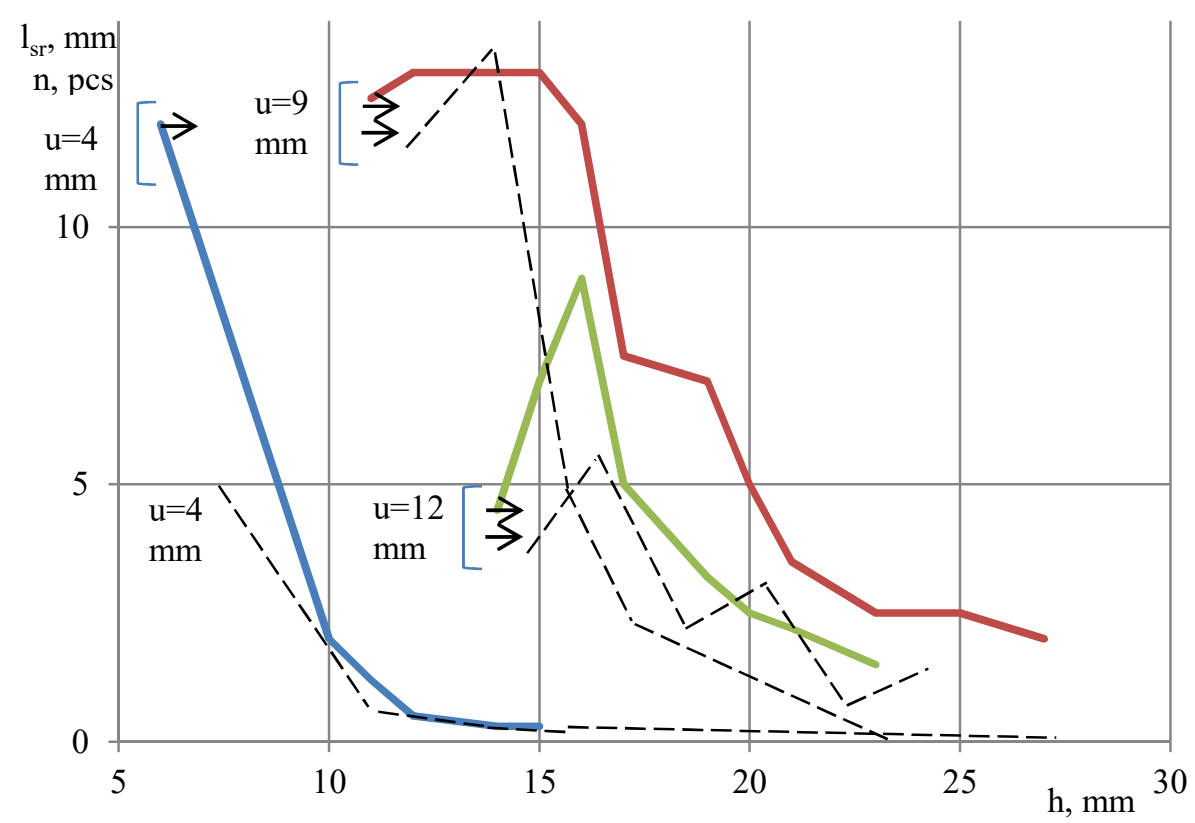

Fig. 3. The number $n$ (solid lines) and the total length $l_{\text {cp }}$ of cracks (dashed lines) in core of crosspieces at different depths $h$

Table 1. Calculated results of average crack lengths

\begin{tabular}{|c|c|c|c|c|c|c|c|c|c|}
\hline \multirow{2}{*}{$\begin{array}{l}\text { Passed cargo, } \\
\text { million tones } \\
\text { gross }\end{array}$} & \multirow{2}{*}{$\begin{array}{c}\text { Cross } \\
\text { piece } \\
\text { wears, } \\
\mathrm{mm}\end{array}$} & \multicolumn{7}{|c|}{$\begin{array}{l}\text { Average length of cracks in cores } \\
\text { of crosspieces at depth of, } \mathrm{mm}\end{array}$} & \multirow{2}{*}{$\begin{array}{c}\text { Average } \\
\text { crack length } \\
\text { over entire } \\
\text { cross } \\
\text { section, mm }\end{array}$} \\
\hline & & 6 & 12 & 14 & 15 & 16 & 20 & 24 & \\
\hline 55 & 4 & 0.21 & 0.30 & 0.20 & 0.05 & 0 & 0 & 0 & 0.21 \\
\hline 78 & 9 & - & 0.50 & 0.35 & 0.19 & 0.19 & 0.14 & 0.11 & 0.24 \\
\hline 186 & 12 & - & - & 0.42 & 0.36 & 0.31 & 0.75 & 0.75 & 0.46 \\
\hline
\end{tabular}

Another important indicator is the conditional probabilities of the appearance of at least one crack at any point in the cross section at a given depth $[17,18,19,20]$. To determine them, we consider the cross section of the core of the crosspiece and a metal layer of unit thickness located at the $i$-th depth from the surface of the core. If the length of the entire layer is $L_{i}$, and the total length of the cracks in this layer is $\sum l_{i}$, then the probability that there will be a crack at any point in the layer can be found as the ratio of the fraction of points at which there is damage to the total number of points layer: $P_{i}=\sum l_{i} / L_{i}$. The conditional probabilities calculated using this formula are given in table. 2. They also characterize the fraction of the cross section at a given depth damaged by cracks. From the data table. Figure 2 shows that as the volume of passed cargo (wear) increases at almost all depths from the surface, the number of points at which previously occurred cracks occur or develop grows, which indicates the presence of a direct dependence of the increase in damage to the core metal on the value of the cargo passed through the crosspiece.

The data in table 2 can serve only for a qualitative assessment of ongoing processes, since they do not have sufficient statistical representativeness [21, 22]. This, obviously, explains the fact that in a layer at a depth of $14 \mathrm{~mm}$ from an unworn surface, a crosspiece with a wear of $12 \mathrm{~mm}$, the probability of damage was less than a cross with a wear of $9 \mathrm{~mm}$. 
However, the general tendency to increase the proportion of the section damaged by defects with increasing wear (operating time) is not in doubt.

Table 2. Conditional probabilities of cracks at points of core layer

\begin{tabular}{|c|c|c|c|c|c|c|c|}
\hline \multirow{2}{*}{$\begin{array}{c}\text { Passed cargo, } \\
\text { million tones gross }\end{array}$} & \multirow{2}{*}{$\begin{array}{c}\text { Crosspiece } \\
\text { wears, mm }\end{array}$} & \multicolumn{6}{|c|}{$\begin{array}{c}\text { Conditional probabilities of presence of cracks at points } \\
\text { of core layer located at depth, mm }\end{array}$} \\
\cline { 3 - 8 } & & 6 & 12 & 14 & 16 & 20 & 24 \\
\hline 55 & 4 & 0.16 & 0.015 & 0.001 & 0 & 0 & 0 \\
\hline 78 & 9 & - & 0.380 & 0.290 & 0.11 & 0.04 & 0.02 \\
\hline 186 & 12 & - & - & 0.110 & 0.17 & 0.08 & 0.04 \\
\hline
\end{tabular}

\section{Conclusions}

1. The change in the hardness of metal along the depth of cross-section of the crosspiece at the stage of steady wear occurs with the wear rate of the crosspiece.

2. From the surface of the crosspiece into the depth of the cross section, the hardness of the metal decreases. The rule of change can be considered linear.

3 . The generalized results of studies of the susceptibility to metal defects of the crosspieces showed that the processes of occurrence and development of contact defects on the joints are fatigue-like.

\section{References}

1. B. Glusberg, V. Korolev et al. MATEC Web of Conferences, 239, 01054 (2018) DOI: 10.1051/matecconf/201823901054

2. B. Glusberg, V. Korolev et al. E3S Web of Conferences, 138, 01017 (2019) DOI: $10.1051 / \mathrm{e} 3$ sconf $/ 201913801017$

3. E. Gridasova, P. Nikiforov, A. Loktev et al. TransSiberia 2019: VIII International Scientific Siberian Transport Forum, 559-569 DOI: 10.1007/978-3-030-37916-2_54

4. A.A. Loktev, V.V. Korolev, E.A. Gridasova. IOP Conf. Series: Materials Science and Engineering, 687, 022036 (2019) DOI: 10.1088/1757-899X/687/2/022036

5. B. Glusberg, A. Loktev et al. Advances in Intelligent Systems and Computing, 982, 337-345 (2020) DOI: 10.1007/978-3-030-19756-8_31

6. A. Lyudagovsky, A. Loktev, V. Korolev et al. E3S Web of Conferences, 110, 01017 (2019) DOI: $10.1051 / \mathrm{e} 3$ sconf $/ 201911001017$

7. A. Loktev, V. Korolev et al. Procedia Engineering, 189, 133-137 (2017) DOI: 10.1016/j.proeng.2017.05.022

8. A. Loktev, V. Korolev et al. Vestnik of the Railway Research Institute, 77(2), 77-83 (2018) DOI: 10.21780/2223-9731-2018-77-2-77-83

9. A. Loktev, V. Korolev et al. IOP Conf. Series: Materials Science and Engineering, 463, 032018 (2018) DOI: 10.1088/1757-899X/463/3/032018

10. B. Glusberg, A. Savin, A. Loktev et al. Advances in Intelligent Systems and Computing, 982, 556-570 (2020) DOI: 10.1007/978-3-030-19756-8_53

11. B. Glusberg, A. Savin, A Loktev et al. Advances in Intelligent Systems and Computing, 982, 571-587 (2020) DOI: 10.1007/978-3-030-19756-8_54

12. V. Korolev. TransSiberia 2019: VIII International Scientific Siberian Transport Forum, 621-638 DOI: 10.1007/978-3-030-37916-2_60 
13. A. Loktev, V. Korolev et al. IOP Conf. Series: Materials Science and Engineering, 463, 032019 (2018) DOI: 10.1088/1757-899X/463/3/032019

14. A. Savin, A. Kogan et al. International Journal of Innovative Technology and Exploring Engineering, 7, 2325-2328 (2019)

15. A. Savin, O. Suslov et al. TransSiberia 2019: VIII International Scientific Siberian Transport Forum, 648-654 DOI: 10.1007/978-3-030-37916-2_62

16. A. Savin, V. Korolev et al. TransSiberia 2019: VIII International Scientific Siberian Transport Forum, 797-808 DOI: 10.1007/978-3-030-37916-2_78

17. A. Loktev, V. Korolev et al. Advances in Intelligent Systems and Computing, 982, 325-336 (2020) DOI: 10.1007/978-3-030-19756-8_30

18. A.V. Savin, V.V. Korolev, I.V. Shishkina. IOP Conf. Series: Materials Science and Engineering, 687, 022035 (2019) DOI: 10.1088/1757-899X/687/2/022035

19. A. Loktev, V. Korolev et al. TransSiberia 2019: VIII International Scientific Siberian Transport Forum, 209-218 (2020) DOI: 10.1007/978-3-030-37919-3_20

20. A. Loktev, V. Korolev et al. Vestnik of the Railway Research Institute, 77(6), 331-336 (2018) DOI: 10.21780/2223-9731-2018-77-6-331-336

21. V. Korolev. TransSiberia 2019: VIII International Scientific Siberian Transport Forum, 175-187 DOI: 10.1007/978-3-030-37919-3_17

22. V. Korolev, A. Loktev et al. IOP Conference Series: Earth and Environmental Science, 403, DOI: 10.1088/1755-1315/403/1/012194 